\title{
Stereocontrolled Synthesis of Tungsten- $\pi$-Allyl Complexes Comprising Two Remoted Alcohols
}

\author{
J ang-Shyang Fang, ${ }^{\dagger}$ Gene-Hsian Lee, ${ }^{\ddagger}$ Shie-Ming Peng, ${ }^{\ddagger}$ and Rai-Shung Liu*,† \\ Department of Chemistry, National Tsing-Hua University, Hsinchu, 30043, \\ Taiwan, Republic of China, and Department of Chemistry, National Taiwan University, \\ Taipei, 10764, Taiwan, Republic of China
}

Received J une 27, 2000

\begin{abstract}
Organic lithium reagents add to the carbonyl group of $\mathrm{CpW}(\mathrm{CO})_{2}\left(\eta^{3}-\gamma\right.$-lactonyl $\mathbf{4 a}$ to give the $\pi$-allyl $\eta^{3}$-hydroxycarbonyl tungsten complexes $\mathbf{5 a - 5 d}$ with good yields (79-94\%). An $X$-ray study of compound 5 a revealed the acetyl group has $\pi$-del ocalization with a tungsten$\pi$-allyl group. A subsequent reaction of 5 a with $\mathrm{DIBAL}-\mathrm{H}, \mathrm{RMgBr}(\mathrm{R}=\mathrm{Ph}, \mathrm{Et})$, and ${ }^{\mathrm{n} B u L i}$ gave the diols $\mathrm{CpW}(\mathrm{CO})_{2}\left[\left(3 \mathrm{R} *, 4 \mathrm{~S}^{*}\right)-\pi-2-\mathrm{CMe}(\mathrm{OH}) \mathrm{R}-\mathrm{CH}_{2} \mathrm{CCHCMe}(\mathrm{OH})\right](\mathrm{R}=\mathrm{H} \mathbf{6 a}, \mathrm{Ph} \mathbf{6 b}$, Et $\mathbf{6 c}^{\mathrm{c}},{ }^{\mathrm{n}} \mathrm{Bu} \mathbf{6 d}$ ) as a single diastereomer; the yields were $64 \%-86 \%$. The acyl group of $\mathrm{CpW}-$ $(\mathrm{CO})_{2}\left[\left(3 \mathrm{R} *, 4 \mathrm{~S}^{*}\right)-\pi-2-\mathrm{COMe}-\mathrm{CH}_{2} \mathrm{CCHCMe}(\mathrm{OTBS})\right](7)$ was deprotonated by LDA at $-78{ }^{\circ} \mathrm{C}$ to form an enolate that condensed with $\mathrm{RCHO}\left(\mathrm{R}={ }^{\mathrm{i}} \mathrm{Pr}\right.$, $\left.{ }^{\mathrm{i}} \mathrm{Bu}\right)$ to form a pair of aldol products $\mathbf{8} \mathbf{a}, \mathbf{8 b}$ and $\mathbf{9 a}, \mathbf{9 b}$, which were separated by column chromatography. In the presence of $\mathrm{n}_{-}$ $\mathrm{Bu}_{2} \mathrm{BOTf}$, the diastereosel ectivities of $\mathbf{8 b}$ and $\mathbf{9 b}$ were improved significantly. The structures of these aldol products were elucidated by X-ray diffraction study of the representative product $\mathbf{8 b}$.
\end{abstract}

\section{Introduction}

Nucleophilic addition of a $\mathrm{C}=\mathrm{X}$ group $(\mathrm{X}=\mathrm{O}, \mathrm{NR})$ adjacent to a metal fragment $M L_{n}$ normally proceeds with high diastereosel ectivities due to steric and electronic effects. ${ }^{1,2}$ Sterically, a metal fragment can block nucleophilic attack from the metal side, and this phenomenon allows only anti-addition of nucleophiles. ${ }^{1-3}$ For electron-rich metal fragments, ${ }^{1-3}$ the orbital overlap between the metal fragment and the $\mathrm{C}=\mathrm{X}$ group is significant, and in most cases only one confomer exists predominantly in both solution and solid structure. Scheme 1 (eq 1) shows an instance where the s-cisconformer is the observed species for $\mathrm{CpMo}(\mathrm{CO})_{2}(\pi-1-$ $\left.\mathrm{C}_{3} \mathrm{H}_{4} \mathrm{COMe}\right)^{3 b}(\mathbf{1})$ in solution, and its reaction with $\mathrm{NaBH}_{4}$ and Grignard reagents gave only one stereoisomeric product. The resulting $\eta^{3}$-allyl alcohol (2) is subsequently transformed into various 1,3-diols on further functionalization. ${ }^{3 \mathrm{~b}}$ Recently, we reported an acid-catalyzed intramolecular carbonylation of tungstenpropargyl species (3) (eq 2), leading to a tungsten-synbutyrolactonyl compound (4). 4,5 As a continuing work on functionalization of tungsten-allyl complexes, we report stereocontrolled synthesis of acydic diols starting

\footnotetext{
† National Tsing-Hua University.
}

‡ National Taiwan University.

(1) (a) Gree, R. Synthesis 1989, 341. (b) Wada, C. K.; Roush, W. R. Tetrahedron Lett. 1994, 35, 7351. (c) Uemura, M. In Advances in MetalOrganic Chemistry; 1991; Vol. 2, p 1.

(2) (a) Uemura, M.; Minami, T.; Yamashita, Y.; Hiyoshi, K. i.; Hayashi, Y. Tetrahedron Lett. 1987, 28, 641. (b) Harvey, D. F.; Selchau, V. B. J. Org. Chem. 2000, 65, 2282.

(3) (a) Liao, M, -F.; Lee, G.-H.; Peng, S. M.; Liu, R. S. Organome tallics 1994, 14, 4973. (b) Vong, W.-J .; Peng, S.-M.; Lin, S.-S.; Lin, W.J .; Liu R.-S. J . Am. Chem. Soc. 1991, 113, 573.

(4) Chen, C.-C.; Fan, J. S.; Lee, G. H.: Peng, S. M.; Wang, S. L.; Liu, R. S. J . Am. Chem. Soc. 1995, 117, 2933.

(5) Chen, C.-C.; Fan J .-S.; Shieh, S.-J .; Lee G.-H.; Peng S.-M.; Wang S.-L.; Liu, R.-S. J . Am. Chem. Soc. 1996, 118, 9279.

\section{Scheme 1}

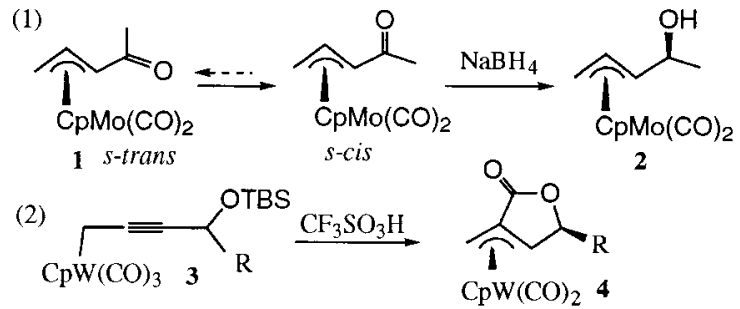

from tungsten $\pi$-butyrolactone complex 4 . Stereocontrolled synthesis of an acyclic molecule comprising two remoted alcohols is an intriguing topic in synthetic chemistry.

\section{Results and Discussions}

Shown in Scheme 2 is ring-opening of compound $\mathbf{4 a}$ effected by various organic lithium reagents to afford the al cohols $\mathbf{5} \mathbf{a}-\mathbf{5 d}$. The molecular structure of $\mathbf{5} \mathbf{a}^{6}$ was characterized by an X-ray diffraction study (Figure 1). The acetyl group is quite coplanar with the allyl fragment, and the dihedral angle between the two planes is ca. $4.5^{\circ}$. The $v(\mathrm{CO})$ frequency of the acetyl group is observed at $1660 \mathrm{~cm}^{-1}$. This information suggests that the acetyl group forms partial $\pi$-overlap with its $\pi$-allyl group. Compound $\mathbf{5 a}$ also retains the same conformation in solution according to its proton NOE NMR spectra. With the tungsten- $-\pi$-allyl fragment as a stereotemplate, compound $\mathbf{5 a}$ is transformed into acyclic diols $\mathbf{6 a}-\mathbf{6 d}$ in the reactions with DIBAL-H, $\mathrm{RMgBr}(\mathrm{R}=\mathrm{Ph}, \mathrm{Et})$, and $\mathrm{RLi}(\mathrm{R}=\mathrm{nBu})$. The reactions proceed with excellent diastereoselectivities to give one

(6) Crystal data for 5a: tetragonal P-421c, a $=13.988(5) \AA$, c $=$ 14.338(7) $\AA, V=2809.4(17) A^{3}, Z=8$, final $R=0.025$ and $R_{w}=0.0025$ for 1210 observed reflections $(|>2 \sigma|)$ out of 1396. 

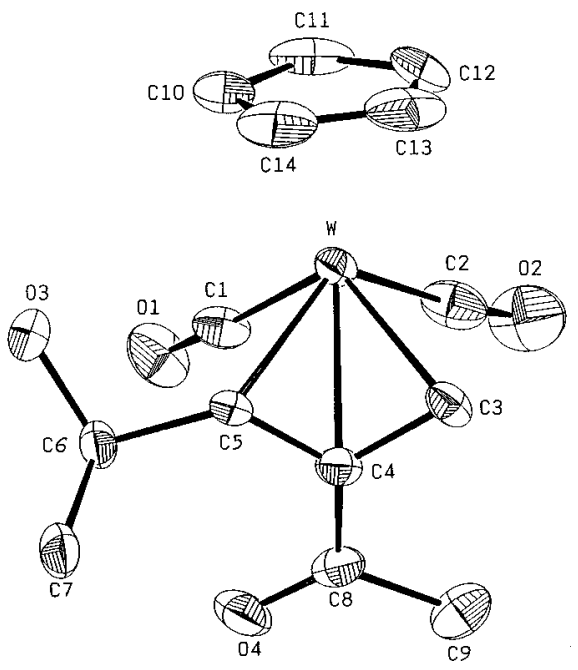

Figure 1. Molecular structure of compound 5a. Selected bond distances $(\AA)$ : $C(3)-C(4)$ 1.434(15), C(4)-C(5) $1.437(14), C(4)-C(8) 1.490(16), C(8)-O(4) 1.216(15), C(6)-$ $\mathrm{O}(3)$ 1.436(14).

\section{Scheme 2}

(1)<smiles>C[C@@H]1OC(=O)C2=C[C@H]1C[C@H]2[N+](=O)[O-]</smiles><smiles>[R]OC(=O)C(C[C@@H](C)O)C(=O)[N+](=O)[O-]</smiles>
$R=\operatorname{Me}(5 a) 94 \%,{ }^{n} \mathrm{Bu}(5 \mathrm{c}) 79 \%$ Ph (5b) $81 \%$, vinyl (5d) $81 \%$

(2)

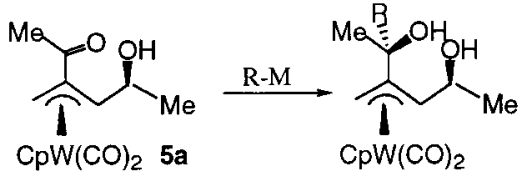

\begin{tabular}{|c|l|l|}
\cline { 2 - 3 } entry & $\mathrm{R}-\mathrm{M}$ & \multicolumn{1}{|c|}{ yields } \\
\hline 1 & $\mathrm{DIBAL}-\mathrm{H}$ & $\mathrm{R}=\mathrm{H}(\mathbf{6 a}) 65 \%$ \\
2 & $\mathrm{PhMgBr}$ & $\mathrm{R}=\mathrm{Ph}(\mathbf{6 b}) 85 \%$ \\
3 & ${ }^{\mathrm{n}} \mathrm{BuLi}$ & $\mathrm{R}=\mathrm{B}_{\mathrm{Bu}}(\mathbf{6 c}) 68 \%$ \\
4 & $\mathrm{EtMgBr}$ & $\mathrm{R}=\mathrm{Et}(\mathbf{6 d}) 68 \%$ \\
\hline
\end{tabular}

diastereomeric product in $65-86 \%$ yield. Clarification of the structures of $\mathbf{6 a}-\mathbf{6 d}$ relies on X-ray diffraction studies of the representative compound $\mathbf{6 a}$ (F igure 2$){ }^{7}$ The ORTEP drawing reveals that compound $\mathbf{6 a}$ is produced via attack of hydride at the ketone group of $5 \mathbf{a}$ anti to the tungsten- $\pi$-allyl fragment.

We also examined the aldol reaction of the tertbutyldimethylsilyloxy derivative 7 that was prepared in $89 \%$ yield from its parent alcohol $\mathbf{5 a}$. The enolate of compound 7 was generated by LDA at $-78^{\circ} \mathrm{C}$ in THF, and its reaction with al dehydes $\mathrm{RCHO}\left(\mathrm{R}={ }^{\mathrm{i}} \mathrm{Pr},{ }^{\mathrm{i}} \mathrm{Bu}\right)$ at this temperature afforded two aldol products $\left(R={ }^{i} \mathrm{Pr}\right.$, $\mathbf{8 a}$ and $\mathbf{8 b}$; $\mathrm{R}={ }^{\mathrm{i}} \mathrm{Bu}, \mathbf{9 a}$ and $\mathbf{9 b}$ ), respectively. These two diastereomers were separable on a silica column, and their isolated yields are shown in Scheme 3 (entries 1 and 3). Compounds $\mathbf{8 a}$ and $\mathbf{9 a}$ are distinguishable from $\mathbf{8 b}$ and $\mathbf{9 b}$ in their proton NMR spectra. The $\mathrm{CH}_{2}$ protons of $\mathbf{8 a}$ and $9 \mathbf{a}$ show an $A B$ pattern in the ${ }^{1} \mathrm{H}$

(7) Crystal data for 6a: monodinic P21/c, a = 7.789(5) $\AA, b=25.508$ (8) $\AA, c=14.817(4) \AA, V=2931.1(22) A^{3}, Z=8$, final $R=0.035$ and $\mathrm{R}_{\mathrm{w}}=0.035$ for 3650 observed reflections $(I>2 \sigma \mathrm{l})$ out of 5146 reflections.
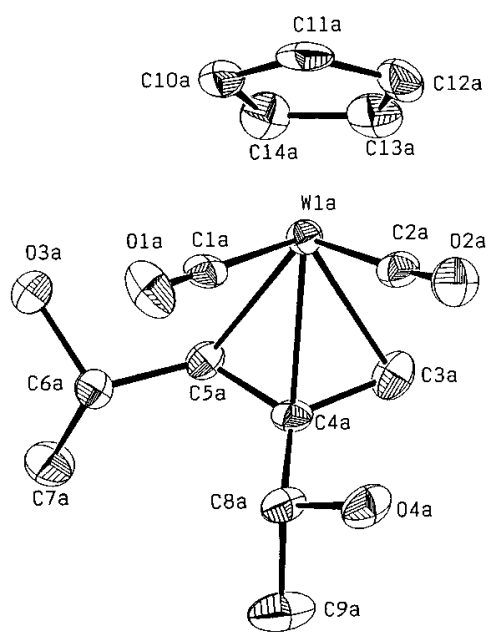

Figure 2. Molecular structure of compound 6a. Selected bond distances $(\AA)$ : $C(3 a)-C(4 a) 1.453(15), C(4 a)-C(5 a)$ 1.438(13), C(4a)-C(8a) 1.513(14), C(8a)-O(4a) 1.411(12),

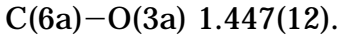

\section{Scheme 3}

(1)

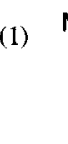

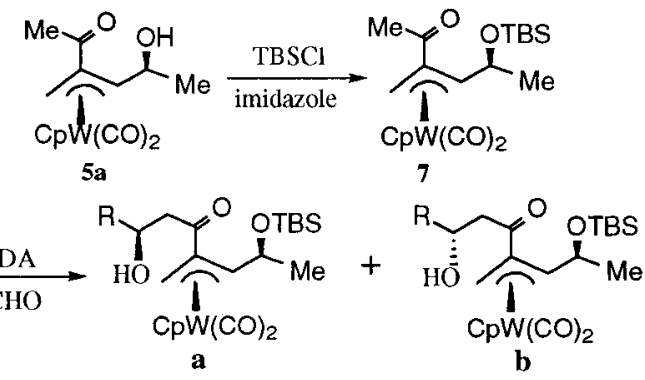

\begin{tabular}{|cccc|}
\hline entry & RCHO & L.A. & Yields \\
\hline $\mathbf{1}$ & ${ }^{\mathrm{i}} \mathrm{PrCHO}$ & - & $\mathbf{8 a}(37 \%), \mathbf{8 b}(45 \%)$ \\
$\mathbf{2}$ & ${ }^{\mathrm{i}} \mathrm{PrCHO}$ & $\mathrm{Bu}_{2} \mathrm{BOTf}$ & $\mathbf{8 a}(19 \%), \mathbf{8 b}(61 \%)$ \\
$\mathbf{3}$ & ${ }^{\mathrm{i}} \mathrm{BuCHO}$ & - & $\mathbf{9 a}(33 \%), \mathbf{9 b}(50 \%)$ \\
$\mathbf{4}$ & ${ }^{\mathrm{B}} \mathrm{BuCHO}$ & $\mathrm{Bu}_{2} \mathrm{BOTf}$ & $\mathbf{9 a}(13 \%), \mathbf{9 b}(70 \%)$ \\
\hline
\end{tabular}

(2)

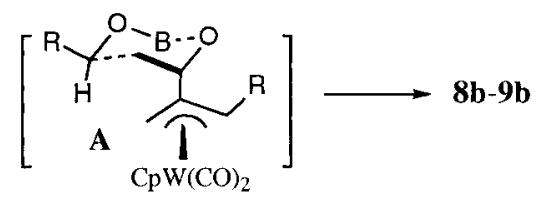

NMR spectra, whereas those of $\mathbf{8 b}$ and $\mathbf{9 b}$ show a simple doublet resonance. The structure of compound $\mathbf{8 b}$ was determined by X-ray diffraction study (Figure 3). ${ }^{9}$ The aldol diastereoselectivities can be improved significantly by the presence of ${ }^{n} \mathrm{Bu}_{2} \mathrm{BOTf}$, and the results are given in entries 2 and 4 . The diastereomeric ratios of $\mathbf{8 b} / \mathbf{8 a}$ (61/19) and $\mathbf{9 b / 9 a}$ (70/13) were estimated on the basis of the yields of isolated products. The improving effect of ${ }^{n} \mathrm{Bu}_{2} \mathrm{BOTf}$ suggests that the major diastereomers $\mathbf{8 b}$ and $\mathbf{9 b}$ are produced via a chairlike transition state A. ${ }^{8-10}$ Such a transition state was previouly proposed for aldol reactions of molybdenum- $\pi$-allyl enolates. ${ }^{3}$ To realize the synthetic utility of these complexes, com-

(8) Evans, D. A.; Vogel, E.; Nelson, J . V. J . Am. Chem. Soc. 1979, $101,6120$.

(9) Heathcock, C. H.; Buse, C. T.; Kleschick, W. A.; Pirrung, M. C.; Sohn, J . E..; Lampe, J. J . Org. Chem. 1980, 45, 1066.

(10) Fellman, P.; Dubois, J. E. Tetrahedron 1978, 34, 1349. 


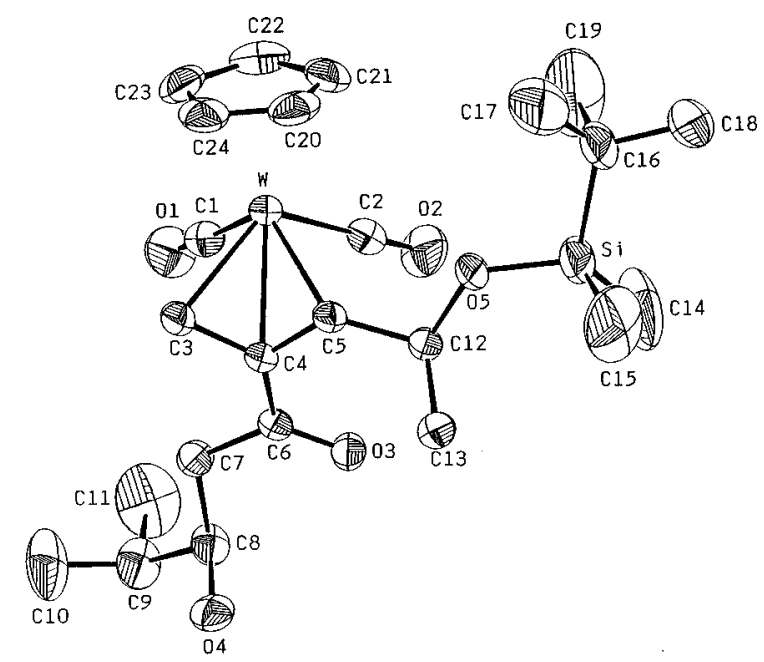

Figure 3. Molecular structure of compound $\mathbf{8 b}$. Selected bond distances $(\AA)$ : $C(3)-C(4) 1.413(7), C(4)-C(5) 1.417-$ (7), $\mathrm{C}(4)-\mathrm{C}(6) 1.496(7), \mathrm{C}(6)-\mathrm{O}(3) 1.220(6), \mathrm{C}(8)-\mathrm{O}(4)$ $1.422(7)$.

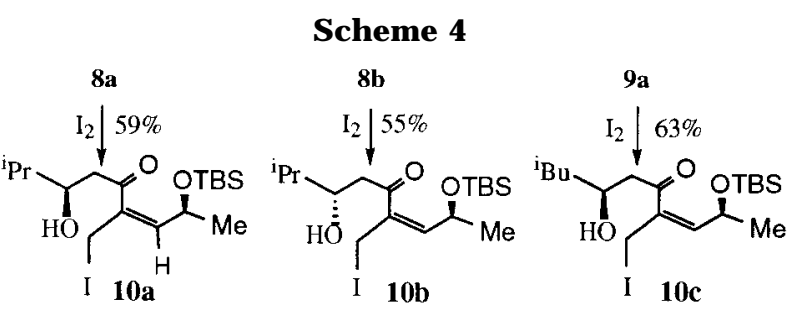

pounds $\mathbf{8 a}, \mathbf{8 b}$ and $\mathbf{9 a}$ were demetal ated smoothly by $\mathrm{I}_{2}$ (1.0-1.1 equiv) in $\mathrm{CH}_{2} \mathrm{Cl}_{2}$ to afford the iodoallyl derivatives 10a-10c in 55-63\% yield (Scheme 4). The structures of $\mathbf{1 0 a}, \mathbf{1 0 b}$ were determined by proton NOE NMR spectra. Irradation of the $\mathrm{CHH}^{\prime} \mathrm{I}$ signal ( $\left.\delta 4.33 \mathrm{ppm}\right)$ of 10a gives an increase in the intensity of the $=\mathrm{CH}$ proton ( $\delta 6.32$ ppm) by $2.0 \%$. In contrast with $\mathbf{8 a}, \mathbf{9 b}$, and $\mathbf{9 a}$, $\mathrm{I}_{2}$-oxidative demetalation of the $\pi$-allyl diols $\mathbf{6 a}-\mathbf{6 d}$ gave a complicated mixture of products. This may be attributed to the vulnerability of the central alcohol, which easily undergoes ionization to form a tungsten $-\eta^{4}$ trimethylenemethane cation in acidic medium. ${ }^{12}$ Treatment of $\mathbf{8 a}, \mathbf{8 b}$ and $\mathbf{9 a}$ with $\mathrm{Ce}(\mathrm{IV})$-salt, $\mathrm{NOBF}_{4} /$ PhSNa, ${ }^{13,14}$ and $\mathrm{NOBF}_{4} / \mathrm{NaBH}_{4}$ was al so unsuccessful.

A metal fragment comprising two stereogenic alcohols, amines, and phosphines can be utilized as a ligand. ${ }^{15}$ Compound $\mathbf{4 a}$ can be prepared in enantiomerically pure form via chiral chloropropargyl alcohol.4,5 In principle, chiral tungsten- $\pi$-allyl complexes having diols, diamines, and diphosphines can be prepapred using the allyl diols $\mathbf{6 a}-\mathbf{6 d}$ as starting materials. These chiral compounds may be useful as a ligand in asymmetric catalysis. Studies toward this direction are currently underway.

(11) Crystal data for $\mathbf{8 b}$ : triclinic $P \overline{1}, a=8.554(3) \AA, b=11.988(3)$ $\AA, C=13.859(4) \AA, V=1368.2(7) \mathrm{A}^{3}, Z=2$, final $R=0.030$ and $R_{w}=$ 0.032 for 4264 observed reflections $(I>2 \sigma \mid)$ out of 4820 reflections. (12) Su G.-M.; Lee G.-H.; Peng, S.-M.; Liu R.-S. J . Chem. Soc., Chem. Commun. 1992, 215

(13) (a) Liebeskind, L. S.; Bombrum, A. J . Am. Chem. Soc. 1991 113, 8736. (b) Pearson, A. J . Blyston, S. L. Nar, H.; Pinkerton, A. A.; Roden, B. A.; Yoon, J. J . Am. Chem. Soc. 1989, 111, 134.

(14) Faller, J. W.; Rosan, A, M. J . Am. Chem. Soc. 1976, 98, 3388.

(15) (a) Togni, A.; Breutel, C.; Schnyder, A.; Spindler, F.; Landert, H.; Tijani, A. J . Am. Chem. Soc. 1994, 116, 4062. (b) Togni, A. Angew. Chem., Int. Ed. Engl. 1996, 35, 1475. (c) Zanetti, N. C.; Spindler, F.; Spencer, J.; Togni, A.; Rihs, G. Organometallics 1996, 15, 861.

\section{Experimental Section}

Unless otherwise noted, all reactions were carried out under nitrogen atmosphere in oven-dried glassware using standard syringe, cannula, and septa apparatus. Benzene, diethyl ether, tetrahydrofuran, and hexane were dried with sodium benzophenone and distilled before use. Dichloromethane was dried over $\mathrm{CaH}_{2}$ and distilled before use. W(CO) $)_{6}$, sodium, dicyclopentadiene, propargyl bromide, methanesulfonamide, p-toluenesulfonamide, and benzaldehyde were obtained commercially and used without purification. Spectral data of compound 4a were published previously. ${ }^{5}$

Reaction of MeLi with 4 a. To a THF solution (12 mL) of compound 4 a $(1.00 \mathrm{~g}, 2.40 \mathrm{mmol})$ was added MeLi (1.6 M, 1.60 $\mathrm{mL}$, hexane) at $-78{ }^{\circ} \mathrm{C}$, and the mixture was stirred for $2 \mathrm{~h}$ before addition of a saturated $\mathrm{NH}_{4} \mathrm{Cl}$ solution $(2.0 \mathrm{~mL})$. The organic layer was extracted with diethyl ether and eluted through a silica column (diethyl ether/hexane $=1 / 1$ ) to give compound 5a $(0.97 \mathrm{~g}, 2.25 \mathrm{mmol}, 94 \%)$ as a yellow solid. IR $\left(\mathrm{CH}_{2} \mathrm{Cl}_{2}\right): v$ (CO) 1969 (vs), 1897 (vs), 1661 (m) cm ${ }^{-1} .{ }^{1} \mathrm{H}$ NMR $\left(300 \mathrm{MHz} \mathrm{CDCl}_{3}\right): \delta 5.35(\mathrm{~s}, 5 \mathrm{H}), 4.85(\mathrm{~m}, 1 \mathrm{H}), 2.54(\mathrm{~s}, 1 \mathrm{H})$, $2.12(\mathrm{~s}, 4 \mathrm{H}), 1.33(\mathrm{~d}, \mathrm{~J}=6.0 \mathrm{~Hz}, 3 \mathrm{H}), 1.11(\mathrm{~s}, 1 \mathrm{H}) .{ }^{13} \mathrm{C} N M R$ $\left(75 \mathrm{MHz} \mathrm{CDCl}_{3}\right): \delta 224.4 ; 220.2,201.8,93.7,88.1,83.9,67.8$, $61.8,25.8,25.4,21.5$. Mass (EI, $75 \mathrm{eV}): 432\left(\mathrm{M}^{+}\right)$. Anal. Calcd for $\mathrm{C}_{13} \mathrm{H}_{14} \mathrm{WO}_{4}$ : C 38.91; $\mathrm{H}$ 3.73. Found: C 38.82; $\mathrm{H}$ 3.77.

Synthesis of Compound $\mathbf{5 b}$. This compound was prepared similarly from the reaction between $\mathbf{4 a}$ and $\mathrm{PhLi}$. The crude product was purified by column chromatography (silica, diethyl ether/hexane $=1 / 1$ ) to afford $\mathbf{5 b}$ as a yellow solid ( $81 \%$ yield). IR (neat): $v$ (CO) 1964 (vs); 1895 (vs); 1620 (m) cm ${ }^{-1} .{ }^{1} \mathrm{H} ~ N M R$ $\left(300 \mathrm{MHz} \mathrm{CDCl}_{3}\right): \delta 7.71-7.33(\mathrm{~m}, 5 \mathrm{H}), 5.31(\mathrm{~s}, 5 \mathrm{H}), 4.98(\mathrm{~m}$, $1 \mathrm{H}), 2.56(\mathrm{~s}, 1 \mathrm{H}), 2.31(\mathrm{~d}, \mathrm{~J}=6.2 \mathrm{~Hz}, 1 \mathrm{H}), 1.36(\mathrm{~d}, \mathrm{~J}=6.0 \mathrm{~Hz}$, $3 \mathrm{H}), 1.24(\mathrm{~s}, 1 \mathrm{H}) .{ }^{13} \mathrm{C}$ NMR $\left(75 \mathrm{MHz} \mathrm{CDCl}_{3}\right): \delta 221.4 ; 221.1$, 199.5, 139.5, 131.7, 128.6, 127.8, 87.9, 83.4, 68.0, 63.0, 25.6, 24.3. Mass $\left(\mathrm{El}^{+}, 75 \mathrm{eV}\right): 494\left(\mathrm{M}^{+}\right)$. Anal. Calcd for $\mathrm{C}_{19} \mathrm{H}_{18^{-}}$ $\mathrm{WO}_{4}$ : C 46.18; H 3.67. Found: C 45.92; H 3.71.

Synthesis of Compound $\mathbf{5 c}$. The reaction of $\mathbf{4 a}$ and BuLi, followed by chromatographic purification (silica, diethyl ether/ hexane $=1 / 1)$, gave $5 \mathbf{c}$ as a yellow solid $(79 \%$ yield $)$. I $R\left(\mathrm{CH}_{2^{-}}\right.$ $\mathrm{Cl}_{2}$ ): $v$ (CO) 1967 (vs), 1899 (vs); 1659 (m) cm ${ }^{-1} .{ }^{1} \mathrm{H}$ NMR (300 $\left.\mathrm{MHz} ; \mathrm{CDCl}_{3}\right): \delta 5.35(\mathrm{~s}, 5 \mathrm{H}), 4.89(\mathrm{~m}, 1 \mathrm{H}), 2.57(\mathrm{~s}, 1 \mathrm{H}), 2.46-$ $2.20(\mathrm{~m}, 4 \mathrm{H}), 2.12(\mathrm{~d}, \mathrm{~J}=6.4 \mathrm{~Hz}, 1 \mathrm{H}), 1.59-1.54(\mathrm{~m}, 2 \mathrm{H})$, $1.32-1.22(\mathrm{~m}, 2 \mathrm{H}), 1.36(\mathrm{~d}, \mathrm{~J}=6.4 \mathrm{~Hz}, 3 \mathrm{H}), 1.23(\mathrm{~s}, 1 \mathrm{H}), 0.89$ $(\mathrm{t}, \mathrm{J}=7.4 \mathrm{~Hz}) .{ }^{13} \mathrm{C} N M R\left(75 \mathrm{MHz} \mathrm{CDCl}_{3}\right): \delta 224.4 ; 220.4$, 204.4, 88.1, 83.8, 67.9, 61.9, 37.2, 26.7, 25.3, 22.3, 22.2, 13.9. Mass $\left(\mathrm{El}^{+}, 75 \mathrm{eV} / \mathrm{m}\right)$ : $474\left(\mathrm{M}^{+}\right)$. Anal. Calcd for $\mathrm{C}_{17} \mathrm{H}_{22} \mathrm{WO}_{4}$ : C 43.06; H 4.68. Found. C 43.00; H 4.66.

Synthesis of Compound $\mathbf{5 d}$. The reaction of $\mathbf{4 a}$ with vinyllithium, followed by chromatographic purification (silica, diethyl ether/hexane $=2 / 5)$, gave $\mathbf{5 d}$ as a yellow solid $(81 \%$ yield). IR ( $\mathrm{CH}_{2} \mathrm{Cl}_{2}$ ): $v$ (CO) 1968 (vs), 1898 (vs), 1658 (m) cm ${ }^{-1}$. ${ }^{1} \mathrm{H} \mathrm{NMR}\left(300 \mathrm{MHz} \mathrm{CDCl}_{3}\right): \delta 6.48(\mathrm{~d}, 1 \mathrm{H}, \mathrm{J}=17.2 \mathrm{~Hz}), 6.30$ $(\mathrm{d}, 1 \mathrm{H}, \mathrm{J}=10.2 \mathrm{~Hz}$ ), $5.60(\mathrm{~d}, \mathrm{~J}=17.2 .10 .2 \mathrm{~Hz}, 1 \mathrm{H}), 5.36(\mathrm{~s}$, $5 \mathrm{H}), 4.91(\mathrm{~m}, 1 \mathrm{H}), 2.62(\mathrm{~s}, 1 \mathrm{H}), 2.18(\mathrm{~d}, \mathrm{~J}=6.4 \mathrm{~Hz}, 1 \mathrm{H}), 1.39$ $(\mathrm{d}, \mathrm{J}=6.1 \mathrm{~Hz}, 3 \mathrm{H}), 1.12(\mathrm{~s}, 1 \mathrm{H}) .{ }^{13} \mathrm{C} \mathrm{NMR}\left(75 \mathrm{MHz} \mathrm{CDCl}_{3}\right)$ : $\delta$ 224.9; 221.5, 192.4, 140.7, 128.7, 89.6, 85.0, 68.0, 63.0, 25.6, 22.3. Mass $\left(\mathrm{EI}^{+}, 75 \mathrm{eV}\right): 444\left(\mathrm{M}^{+}\right)$.

Reaction of 5a with DIBAL-H. To a THF (12 mL) solution of 5 a $(0.25 \mathrm{~g}, 0.58 \mathrm{mmol})$ was added DIBAL-H (1.0 M, 0.58 $\mathrm{mL}$ ) at $-78^{\circ} \mathrm{C}$, and the mixture was stirred for $2 \mathrm{~h}$ before it was warmed to $23{ }^{\circ} \mathrm{C}$. The solution was quenched with a saturated $\mathrm{NH}_{4} \mathrm{Cl}$ solution, and the organic layer was extracted with diethyl ether. The extract was dried in vacuo and eluted through a silica column (diethyl ether/hexane $=1 / 1$ ) to give compound $6 \mathrm{a}$ as a yellow solid $(0.16 \mathrm{~g}, 0.37 \mathrm{mmol}, 65 \%)$. IR (neat) $v(\mathrm{CO}) 1960(\mathrm{~s}) ; 1894(\mathrm{~s}) \mathrm{cm}^{-1}$. ${ }^{1} \mathrm{H}$ NMR (300 MHz; $\left.\mathrm{C}_{6} \mathrm{D}_{6}\right)$ : $\delta 4.58(\mathrm{~s}, 5 \mathrm{H}), 4.25(\mathrm{~m}, 2 \mathrm{H}), 2.99(\mathrm{~s}, 1 \mathrm{H}), 2.36(\mathrm{~d}, \mathrm{~J}=9.4 \mathrm{~Hz}$, $1 \mathrm{H}), 1.38(\mathrm{t}, \mathrm{J}=7.4 \mathrm{~Hz}, 3 \mathrm{H}), 1.36(\mathrm{t}, \mathrm{J}=7.4 \mathrm{~Hz}, 3 \mathrm{H}), 0.98(\mathrm{~s}$, $1 \mathrm{H}) .{ }^{13} \mathrm{C}$ NMR (75 MHz; toluene-d 8 ): $\delta$ 219.8; 219.6, 111.3, 89.9, 67.7; 67.2, 61.5, 28.8; 26.1, 24.3. Mass (EI+, 75 eV): 433 
$\left(\mathrm{M}^{+}\right)$. Anal. Calcd for $\mathrm{C}_{14} \mathrm{H}_{18} \mathrm{WO}_{4}$ : C 38.70; $\mathrm{H}$ 4.18. Found: $\mathrm{C}$ 38.0.66; H 4.25.

Synthesis of Compound $\mathbf{6 b}$. The reaction of $\mathbf{5 a}$ and $\mathrm{PhMgBr}$, followed by chromatographic purification (silica, diethyl ether/hexane $=2 / 3)$, gave $\mathbf{6 b}$ as a yellow solid $(85 \%$ yield). IR ( $\mathrm{CH}_{2} \mathrm{Cl}_{2}$ ): v(CO) 1971 (vs), 1899 (vs), 1643 (m) cm ${ }^{-1}$. ${ }^{1} \mathrm{H}$ NMR $\left(300 \mathrm{MHz} \mathrm{CDCl}_{3}\right): \delta 5.33(\mathrm{~s}, 5 \mathrm{H}), 4.92(\mathrm{~m}, 1 \mathrm{H}), 4.02$ $(\mathrm{m}, 1 \mathrm{H}), 2.54(\mathrm{~d}, \mathrm{~J}=1.76 \mathrm{~Hz}, 1 \mathrm{H}), 2.50(\mathrm{~d}, \mathrm{~J}=2.1 \mathrm{~Hz}, 1 \mathrm{H})$, $2.48(\mathrm{~s}, 1 \mathrm{H}), 2.25(\mathrm{~d}, \mathrm{~J}=8.3 \mathrm{~Hz}, 1 \mathrm{H}), 1.78(\mathrm{~m}, 1 \mathrm{H}), 1.44(\mathrm{~m}$, $1 \mathrm{H}), 1.39(\mathrm{~d}, \mathrm{~J}=5.7 \mathrm{~Hz}, 3 \mathrm{H}), 1.11(\mathrm{~m}, 2 \mathrm{H}), 0.89-0.86(\mathrm{br} \mathrm{s}$, $12 \mathrm{H}), 0.09$ (s, 3H); 0.08 (s, 3H). $\left.{ }^{13} \mathrm{C} \mathrm{NMR} \mathrm{(75} \mathrm{M} \mathrm{Hz;} \mathrm{CDCl}_{3}\right): \delta$ 225.3; 220.9, 203.5, 88.6, 83.0, 70.0, 66.4, 62.9, 45.8, 43.9, 27.9, 26.0, 24.3, 23.4; 22.3, 22.0, 17.9, -3.9, -4.2. Mass $\left(\mathrm{EI}^{+}, 75\right.$ eV): $632\left(\mathrm{M}^{+}\right)$. Anal. Calcd for $\mathrm{C}_{25} \mathrm{H}_{40} \mathrm{WSiO}_{5}$ : 47.47; $\mathrm{H} 6.37$. Found: C 47.58; H 6.29 .

Synthesis of Compound $\mathbf{6 c}$. The reaction of BuLi and $\mathbf{5 a}$, followed by chromatographic purification (silica, diethyl ether/ hexane $=2 / 3)$, gave $6 \mathrm{c}$ as a yellow solid $(68 \%$ yield). IR (neat): $v(\mathrm{CO})$ 1967(s); 1892 (s) $\mathrm{cm}^{-1} .{ }^{1} \mathrm{H}$ NMR $(300 \mathrm{MHz}$; $\left.\mathrm{CDCl}_{3}\right): \delta 7.65-7.20(\mathrm{~m}, 5 \mathrm{H}), 5.33(\mathrm{~s}, 5 \mathrm{H}), 4.16(\mathrm{~m}, 2 \mathrm{H}), 3.04$ $(\mathrm{s}, 1 \mathrm{H}), 2.13(\mathrm{~d}, \mathrm{~J}=5.1 \mathrm{~Hz}, 1 \mathrm{H}), 1.73(\mathrm{~s}, 1 \mathrm{H}), 1.49(\mathrm{~s}, 1 \mathrm{H}), 0.74$ $(\mathrm{d}, \mathrm{J}=6.1 \mathrm{~Hz}, 3 \mathrm{H}) .{ }^{13} \mathrm{C} \mathrm{NMR}\left(75 \mathrm{MHz} \mathrm{CDCl}_{3}\right): \delta 219.8 ; 219.6$, $111.3,89.9,67.7 ; 67.2,61.5,28.8 ; 26.1,24.3$. Mass (EI+ 75 eV): $510\left(\mathrm{M}^{+}\right)$. Anal. Calcd for $\mathrm{C}_{20} \mathrm{H}_{22} \mathrm{O}_{4} \mathrm{~W}$ : C 47.08; $\mathrm{H} 4.35$. Found: C 47.86; H 4.54

Synthesis of Compound $\mathbf{6 d}$. The reaction of EtMgBr and 5 a, followed by chromatographic purification (silica, diethyl ether/hexane $=1 / 1)$, gave $\mathbf{6 d}$ as a yell ow solid (68\% yield). IR (neat): $v(\mathrm{CO})$ 1965(s); 1897 (s) $\mathrm{cm}^{-1}$. ${ }^{1} \mathrm{H}$ NMR (300 MHz; $\left.\mathrm{CDCl}_{3}\right): \delta 5.31(\mathrm{~s}, 5 \mathrm{H}), 4.82(\mathrm{~m}, \mathrm{~J}=5.6,4.9 \mathrm{~Hz}, 1 \mathrm{H}), 2.50(\mathrm{~s}$, $1 \mathrm{H}), 2.24(\mathrm{~d}, \mathrm{~J}=4.9 \mathrm{~Hz}, 1 \mathrm{H}), 1.91(\mathrm{~m}, 2 \mathrm{H}), 1.33(\mathrm{~s}, 1 \mathrm{H}), 1.30$ $(\mathrm{d}, \mathrm{J}=5.6 \mathrm{~Hz}, 3 \mathrm{H}), 1.23(\mathrm{~s}, 1 \mathrm{H}), 0.98(\mathrm{t}, \mathrm{J}=7.5 \mathrm{~Hz}, 3 \mathrm{H}) .{ }^{13} \mathrm{C}$ NMR (75 MHz; $\left.\mathrm{CDCl}_{3}\right): \delta 229.7 ; 228.6,110.6,89.1,77.4,67.0$, 58.1, 37.5, 25.8, 25.4, 22.7, 9.9. Mass (EI+ $75 \mathrm{eV}): 462\left(\mathrm{M}^{+}\right)$. Anal. Calcd for $\mathrm{C}_{16} \mathrm{H}_{22} \mathrm{WO}_{4}$ : C 41.58; $\mathrm{H}$ 4.80. Found: $\mathrm{C} 41.93$; H 4.75 .

Synthesis of the tert-Butyldimethylsilyloxy Derivative 7. To a $\mathrm{N}, \mathrm{N}$-dimethylformarmide solution $(20 \mathrm{~mL})$ of $\mathbf{5 a}(5.10$ $\mathrm{g}, 11.8 \mathrm{mmol})$ and imidazole $(1.61 \mathrm{~g}, 23.6 \mathrm{mmol}$ ) was added tert-butyldimethylsilyl chloride $(1.95 \mathrm{~g}, 12.9 \mathrm{mmol})$, and the mixture was stirred for $12 \mathrm{~h}$ before it was quenched with water $(2.0 \mathrm{~mL})$. The organic layer was extracted with diethyl ether $(3 \times 50 \mathrm{~mL})$. The ether extract was dried over $\mathrm{CaCl}_{2}$ and eluted through a silica column (di ethyl ether/hexane $=1 / 2$ ) to afford compound 7 as a yellow solid $(5.72 \mathrm{~g}, 10.5 \mathrm{mmol}, 89 \%)$. I R $\left(\mathrm{CH}_{2^{-}}\right.$ $\mathrm{Cl}_{2}$ ): v(CO) 1968 (vs); 1897 (vs); 1660 (m) cm ${ }^{-1}$. ${ }^{1} \mathrm{H}$ NMR (300 $\left.\mathrm{MHz} \mathrm{CDCl}_{3}\right): \delta 5.32(\mathrm{~s}, 5 \mathrm{H}), 4.91(\mathrm{~m}, 1 \mathrm{H}), 2.57(\mathrm{~d}, \mathrm{~J}=1.6$ $\mathrm{Hz}, 1 \mathrm{H}), 2.31(\mathrm{~d}, \mathrm{~J}=8.4 \mathrm{~Hz}, 1 \mathrm{H}), 1.39(\mathrm{~d}, \mathrm{~J}=5.8 \mathrm{~Hz}, 3 \mathrm{H})$, $1.24(\mathrm{~d}, \mathrm{~J}=1.6 \mathrm{~Hz}, 1 \mathrm{H}), 0.89(\mathrm{~s}, 9 \mathrm{H}), 0.09(\mathrm{~s}, 3 \mathrm{H}), 0.07(\mathrm{~s}, 3 \mathrm{H})$. ${ }^{13} \mathrm{C}$ NMR $\left(75 \mathrm{MHz} \mathrm{CDCl}_{3}\right): \delta 221.4,221.1,199.5,139.5,131.7$, 128.6, 127.8, 87.9, 83.4, 68.0, 63.0, 25.6, 24.3. Mass $\left(\mathrm{EI}^{+}, 75\right.$ eV): $546\left(\mathrm{M}^{+}\right)$. Anal. Calcd for $\mathrm{C}_{20} \mathrm{H}_{30} \mathrm{WSiO}_{4}$ : C 43.97; $\mathrm{H} 5.53$. Found: C 44.06; H 5.54.

Condensation of the Enolate of 7 with 'PrCHO. To a THF solution $(20 \mathrm{~mL})$ of $7(400 \mathrm{mg}, 0.73 \mathrm{mmol})$ was added LDA $(1.6 \mathrm{M}, 6.8 \mathrm{~mL}, 1.1 \mathrm{mmol})$ at $-78^{\circ} \mathrm{C}$, and the mixture was stirred for $1 \mathrm{~h}$ before addition of isopropyl aldehyde (80 $\mathrm{mg}, 1.1 \mathrm{mmol}$ ). The solution was stirred for $2 \mathrm{~h}$ before it was quenched with water. The organic layer was extracted with diethyl ether, dried over $\mathrm{CaCl}_{2}$, and eluted through a silica column (diethyl ether/hexane $=1 / 1)$ to give $8 \mathbf{a}(167 \mathrm{mg}, 0.27$ mmol, 37\%) and $\mathbf{8 b}$ (197 mg, $0.32 \mathrm{mmol}, 45 \%)$, respectively.

Spectral data for 8a: IR $\left(\mathrm{CH}_{2} \mathrm{Cl}_{2}\right) v(\mathrm{CO}) 1971 ; 1901 ; 1644$ $\mathrm{cm}^{-1}{ }^{1}{ }^{1} \mathrm{H}$ NMR $\left(300 \mathrm{MHz} \mathrm{CDCl}_{3}\right) \delta 5.32(\mathrm{~s}, 5 \mathrm{H}), 4.90(\mathrm{~m}, 1 \mathrm{H})$, $3.81(\mathrm{~m}, 1 \mathrm{H}), 2.64(\mathrm{dd}, \mathrm{J}=17.0,2.0 \mathrm{~Hz}, 1 \mathrm{H}), 2.51(\mathrm{~d}, \mathrm{~J}=1.7$ $\mathrm{Hz}), 2.32(\mathrm{dd}, \mathrm{J}=17.0,10 \mathrm{~Hz}, 1 \mathrm{H}), 2.25(\mathrm{~d}, \mathrm{~J}=8.4 \mathrm{~Hz}, 1 \mathrm{H})$, $1.66(\mathrm{~m}, 1 \mathrm{H}), 1.39(\mathrm{~d}, \mathrm{~J}=5.8 \mathrm{~Hz}, 3 \mathrm{H}), 1.10(\mathrm{~d}, \mathrm{~J}=1.7 \mathrm{~Hz}$, $1 \mathrm{H}), 0.89$ (br s, 9H) $0.08(\mathrm{~s}, 3 \mathrm{H}), 0.06(\mathrm{~s}, 3 \mathrm{H}) ;{ }^{13} \mathrm{C}$ NMR $(75$ $\left.\mathrm{MHz} \mathrm{CDCl}_{3}\right) \delta 224.6 ; 220.8,202.9,88.5,83.2,72.5,70.1,63.0$, 40.7, 33.1, 27.9, 26.0, 22.1, 18.4; 17.8, 17.9, -3.9, -4.2; Mass
$\left(\mathrm{EI}^{+}, 75 \mathrm{eV}\right) 618\left(\mathrm{M}^{+}\right)$. Anal. Calcd for $\mathrm{C}_{24} \mathrm{H}_{38} \mathrm{WSiO}_{5}$ : C 46.61; H 6.19. Found. C 46.71; H 6.18.

Spectral data for 8b: IR $\left(\mathrm{CH}_{2} \mathrm{Cl}_{2}\right) v(\mathrm{CO}) 1972$ (vs); 1900 (vs); $1644(\mathrm{~m}) \mathrm{cm}^{-1} ;{ }^{1} \mathrm{H}$ NMR $\left(300 \mathrm{MHz} \mathrm{CDCl}_{3}\right) \delta 5.30(\mathrm{~s}, 5 \mathrm{H}), 4.90$ $(\mathrm{m}, 1 \mathrm{H}), 3.70(\mathrm{q}, \mathrm{J}=5.8 \mathrm{~Hz}, \mathrm{H}), 2.54(\mathrm{~d}, \mathrm{~J}=1.8 \mathrm{~Hz}, 1 \mathrm{H}), 2.48$ $(\mathrm{d}, \mathrm{J}=5.8 \mathrm{~Hz}, 2 \mathrm{H}), 2.25(\mathrm{~d}, \mathrm{~J}=8.3 \mathrm{~Hz}, 1 \mathrm{H}), 1.63(\mathrm{~m}, 1 \mathrm{H})$, $1.36(\mathrm{~d}, \mathrm{~J}=5.8 \mathrm{~Hz}, 3 \mathrm{H}), 1.09(\mathrm{~d}, \mathrm{~J}=1.8 \mathrm{~Hz}, 1 \mathrm{H}), 0.87(\mathrm{br} \mathrm{s}$, $12 \mathrm{H},), 0.07(\mathrm{~s}, 3 \mathrm{H}) ; 0.05(\mathrm{~s}, 3 \mathrm{H}) ;{ }^{13} \mathrm{C} \mathrm{NMR}\left(75 \mathrm{MHz} \mathrm{CDCl}_{3}\right) \delta$ 225.2; 220.9, 203.8, 88.5, 83.1, 72.7, 70.0, 62.9, 40.2, 33.1, 27.9, 25.9, 22.3, 18.3; 17.7, 17.9, -3.9, -4.2; Mass (EI+, $75 \mathrm{eV}) 618$ $\left(\mathrm{M}^{+}\right)$. Anal. Calcd for $\mathrm{C}_{24} \mathrm{H}_{38} \mathrm{WSiO}_{5} \mathrm{C} 46.61 ; \mathrm{H}$ 6.19. Found: $\mathrm{C}$ 46.76; H 6.20.

Aldol Reaction in the Presence of $\mathrm{Bu}_{2} \mathbf{B O T f}$. The enolate generated from LDA and $\mathbf{7}$ was added a THF solution of isopropyl aldehyde ( $80 \mathrm{mg}, 1.1 \mathrm{mmol}$ ) and $\mathrm{Bu}_{2} \mathrm{BOTf}(1.1 \mathrm{mmol})$ at $-78^{\circ} \mathrm{C}$, and the mixture was stirred for $2 \mathrm{~h}$ before it was quenched with water. Workup of the solution gave $\mathbf{8 a}$ and $\mathbf{8 b}$ in $19 \%$ and $61 \%$, respectively.

Syntheses of Compounds 9a and 9b. These two compounds were prepared similarly from the enolate of $\mathbf{7}$ with isobutylaldehyde; the yields of $\mathbf{9 a}$ and $\mathbf{9 b}$ were $32 \%$ and $50 \%$, respectively.

Spectral data for 9a: IR $\left(\mathrm{CH}_{2} \mathrm{Cl}_{2}\right) v(\mathrm{CO}) 1974$ (vs); 1901(vs), $1644(\mathrm{~m}) \mathrm{cm}^{-1}$; ${ }^{1} \mathrm{H}$ NMR (300 MHz; $\left.\mathrm{CDCl}_{3}\right) \delta 5.32(\mathrm{~s}, 5 \mathrm{H})$, $4.90(\mathrm{~m}, 1 \mathrm{H}), 4.10(\mathrm{~m}, 1 \mathrm{H}), 2.64(\mathrm{dd}, \mathrm{J}=17.1,2.5 \mathrm{~Hz}, 1 \mathrm{H})$, $2.50(\mathrm{~d}, \mathrm{~J}=1.7 \mathrm{~Hz}, 1 \mathrm{H}), 2.35(\mathrm{dd}, \mathrm{J}=17.1,8.9 \mathrm{~Hz}, 1 \mathrm{H}), 2.24$ $(\mathrm{d}, \mathrm{J}=8.34 \mathrm{~Hz}, 1 \mathrm{H}), 1.80(\mathrm{~m}, 1 \mathrm{H}), 1.45(\mathrm{~m}, 1 \mathrm{H}), 1.38(\mathrm{~d}, \mathrm{~J}=$ $5.8 \mathrm{~Hz}, 3 \mathrm{H}), 1.09(\mathrm{~m}, 2 \mathrm{H}), 0.88(\mathrm{br} \mathrm{s}, 12 \mathrm{H}), 0.08(\mathrm{~s}, 3 \mathrm{H}) ; 0.06$ $(\mathrm{s} ; 3 \mathrm{H}) ;{ }^{13} \mathrm{C} \mathrm{NMR}\left(75 \mathrm{MHz} \mathrm{CDCl}_{3}\right) \delta 224.7 ; 220.8,202.8,88.5$, 83.0, 70.1, 66.1, 63.0, 45.7, 44.3, 27.9, 26.0, 24.4, 23.4; 22.0, $21.9,17.9,-3.9 ;-4.2 ;$ Mass $\left(\mathrm{El}^{+}, 75 \mathrm{eV}\right) 632\left(\mathrm{M}^{+}\right)$. Anal. Calcd for $\mathrm{C}_{25} \mathrm{H}_{40} \mathrm{O}_{5} \mathrm{SiW}$ : C 47.47; $\mathrm{H}$ 6.37. Found: C 47.40; $\mathrm{H} 6.43$.

Spectral Data for 9b: IR $\left(\mathrm{CH}_{2} \mathrm{Cl}_{2}\right) v(\mathrm{CO}) 1971$ (vs), 1899 (vs), $1643(\mathrm{~m}) \mathrm{cm}^{-1} ;{ }^{1} \mathrm{H}$ NMR $\left(300 \mathrm{MHz} \mathrm{CDCl}_{3}\right) \delta 5.33(\mathrm{~s}, 5 \mathrm{H})$, $4.92(\mathrm{~m}, 1 \mathrm{H}), 4.02(\mathrm{~m}, 1 \mathrm{H}), 2.54(\mathrm{~d}, \mathrm{~J}=1.8 \mathrm{~Hz}, 1 \mathrm{H}), 2.50(\mathrm{~d}, \mathrm{~J}$ $=2.1 \mathrm{~Hz}, 1 \mathrm{H}), 2.48(\mathrm{~s}, 1 \mathrm{H}), 2.25(\mathrm{~d}, \mathrm{~J}=8.3 \mathrm{~Hz}, 1 \mathrm{H}), 1.78(\mathrm{~m}$, $1 \mathrm{H}), 1.44(\mathrm{~m}, 1 \mathrm{H}), 1.39(\mathrm{~d}, \mathrm{~J}=5.7 \mathrm{~Hz}, 3 \mathrm{H}), 1.11(\mathrm{~m}, 2 \mathrm{H}), 0.89$ (s, 3H); $0.86(\mathrm{~s}, 3 \mathrm{H}), 0.09(\mathrm{~s}, 3 \mathrm{H}) ;{ }^{13} \mathrm{C} \mathrm{NMR}\left(75 \mathrm{MHz} \mathrm{CDCl}_{3}\right)$ $\delta$ 225.3; 220.9, 203.5, 88.6, 83.0, 70.0, 66.4, 62.9, 45.8, 43.9, 27.9, 26.0, 24.3, 23.4; 22.3, 22.0, 17.9, -3.9, -4.2; Mass (EI+, $75 \mathrm{eV}) 632\left(\mathrm{M}^{+}\right)$. Anal. Calcd for $\mathrm{C}_{25} \mathrm{H}_{40} \mathrm{WO}_{5} \mathrm{Si}$ : C 47.47; $\mathrm{H} 6.37$. Found: C 47.58; H 6.29.

$\mathbf{I}_{2}$-Demetalation of $\mathbf{8 a}$. To a $\mathrm{CH}_{2} \mathrm{Cl}_{2}$ solution $(5.0 \mathrm{~mL})$ of $8 \mathbf{a}(167 \mathrm{mg}, 0.27 \mathrm{mmol})$ was added $\mathrm{I}_{2}(76.2 \mathrm{mg}, 0.30 \mathrm{mmol})$ at $-40{ }^{\circ} \mathrm{C}$, and the mixture was slowly warmed to $0{ }^{\circ} \mathrm{C}$ over a period of $8 \mathrm{~h}$. To the solution was added $\mathrm{Na}_{2} \mathrm{~S}_{2} \mathrm{O}_{3}(20 \mathrm{mg})$, and the mixture was filtered and concentrated to leave an oil. Elution of this residue over a preparative TLC plate afforded 10a as an oil (49.8 mg, $0.16 \mathrm{mmol}, 59 \%) .{ }^{1} \mathrm{H}$ NMR (300 MHz; $\left.\mathrm{CDCl}_{3}\right): \delta 6.32(\mathrm{~d}, \mathrm{~J}=2.0 . \mathrm{Hz}, 1 \mathrm{H}), 4.33(\mathrm{~d}, \mathrm{~J}=9.0 \mathrm{~Hz}, 1 \mathrm{H})$, $4.23(\mathrm{q}, \mathrm{J}=6 . \mathrm{Hz}, 1 \mathrm{H}), 3.75(\mathrm{~d}, \mathrm{~J}=9.0 \mathrm{~Hz}, 1 \mathrm{H}), 3.48(\mathrm{~m}, 1 \mathrm{H})$, $2.77(\mathrm{~m}, 2 \mathrm{H}), 1.68(\mathrm{~m}, 1 \mathrm{H}), 1.25(\mathrm{~d}, \mathrm{~J}=6.8 \mathrm{~Hz}), 0.94(\mathrm{~d} \mathrm{~J}=$ $6.6 \mathrm{~Hz}, 3 \mathrm{H}), 0.86(\mathrm{~d}, \mathrm{~J}=6.6 \mathrm{~Hz}, 3 \mathrm{H}) .{ }^{13} \mathrm{C} \mathrm{NMR}(75 \mathrm{MHz}$; $\left.\mathrm{CDCl}_{3}\right): \delta 200.8,143.3,137.2,79.3,76.7,50.1,33.3,21.1,18.6$, 18.1, 4.8. Mass $\left(\mathrm{El}^{+}, \mathrm{m} / \mathrm{e}\right)$ : $313\left(\mathrm{M}^{+}\right)$. Anal. Calcd for $\mathrm{C}_{11} \mathrm{H}_{19^{-}}$ $\mathrm{IO}_{3}$ : C, 58.12; $\mathrm{H}, 8.43$. Found: $\mathrm{C}, 58.24 ; \mathrm{H}, 8.43$.

Synthesis of Compound $\mathbf{1 0 b}$. The reaction of $\mathbf{8 b}$ and $I_{2}$, followed by purification on a silica TLC plate, gave $\mathbf{1 0 b}$ as a colorless oil (55\% yield). ${ }^{1} \mathrm{H}$ NMR (300 MHz; $\left.\mathrm{CDCl}_{3}\right): \delta 6.52$ $(\mathrm{d}, \mathrm{J}=3.1 \mathrm{~Hz}, 1 \mathrm{H}), 4.62(\mathrm{dq}, \mathrm{J}=6.8,3.1 \mathrm{~Hz}, 1 \mathrm{H}), 4.04(\mathrm{~s}, 2 \mathrm{H})$, $3.65(\mathrm{~m}, 1 \mathrm{H}), 2.77(\mathrm{~m}, 2 \mathrm{H}), 1.75(\mathrm{~m}, 1 \mathrm{H}), 1.35(\mathrm{~d}, \mathrm{~J}=6.8 \mathrm{~Hz}$, $1 \mathrm{H}), 0.94(\mathrm{~d} J=6.6 \mathrm{~Hz}, 3 \mathrm{H}), 0.86(\mathrm{~d}, \mathrm{~J}=6.6 \mathrm{~Hz}, 3 \mathrm{H}) .{ }^{13} \mathrm{C} \mathrm{NMR}$ $\left(75 \mathrm{MHz}_{;} \mathrm{CDCl}_{3}\right): \delta$ 199.6, 146.1, 139.2, 74.2, 69.3, 48.9, 32.5, 20.5, 18.7, 2.65. Mass (El $\left.{ }^{+}, \mathrm{m} / \mathrm{e}\right)$ : $313\left(\mathrm{M}^{+}\right)$. Anal. Calcd for $\mathrm{C}_{11} \mathrm{H}_{19} \mathrm{O}_{3}$ : C, 58.12; $\mathrm{H}$, 8.43. Found: C, 58.04; $\mathrm{H}, 8.40$.

Synthesis of Compound 10c. The reaction of $9 a$ and $I_{2}$, followed by purification on a silica TLC plate, gave 10c as a colorless oil (63\% yield). ${ }^{1} \mathrm{H}$ NMR $\left(300 \mathrm{MHz} \mathrm{CDCl}_{3}\right): \delta 6.38$ $(\mathrm{d}, \mathrm{J}=2.2 \mathrm{~Hz}, 1 \mathrm{H}), 4.33(\mathrm{~d}, \mathrm{~J}=8.9 \mathrm{~Hz}, 1 \mathrm{H}), 4.29(\mathrm{q}, \mathrm{J}=6.4$ $\mathrm{Hz}, 1 \mathrm{H}), 3.89(\mathrm{~m}, 1 \mathrm{H}), 3.77(\mathrm{~d}, \mathrm{~J}=8.9 \mathrm{~Hz}, 1 \mathrm{H}), 2.76$ 
$(\mathrm{d}, \mathrm{J}=6.7 \mathrm{~Hz}, 2 \mathrm{H}), 1.75(\mathrm{~m}, 1 \mathrm{H}), 1.48(\mathrm{~m}, 1 \mathrm{H}), 1.25(\mathrm{~d}, \mathrm{~J}=6.8$ $\mathrm{Hz}), 1.18(\mathrm{~m}, 1 \mathrm{H}), 0.89(\mathrm{~d}, \mathrm{~J}=6.6 \mathrm{~Hz}, 6 \mathrm{H}) .{ }^{13} \mathrm{C} \mathrm{NMR}(75 \mathrm{MHz}$; $\left.\mathrm{CDCl}_{3}\right): \delta 200.0,144.1,137.5,75.9,72.5,52.5,44.8,24.7,23.2$, 22.3, 21.4, 4.0.44. Mass (El $\left.{ }^{+}, \mathrm{m} / \mathrm{e}\right): 327\left(\mathrm{M}^{+}\right)$.

Acknowledgment. We thank the National Science Council, Republic of China, for financial support of this work.
Supporting Information Available: X-ray data of $\mathbf{5 a}$, $\mathbf{6 a}$, and $\mathbf{8 b}$ including an ORTE P drawing, bond distances and angles, atomic coordinates, and thermal parameters. This material is available free of charge via the Internet at http://pubs.acs.org.

OM000556V 